\title{
Development and clinical application of a new testicular prosthesis
}

\author{
Ye Ning ${ }^{1, *}$, Zhikang Cai ${ }^{2, *}$, Huixing Chen ${ }^{1}$, Ping Ping ${ }^{1}$, Peng $\mathrm{Li}^{1}$, Zhong Wang ${ }^{2}$ and Zheng $\mathrm{Li}^{1}$
}

A new type of testicular prosthesis made of silastic with an elliptical shape to mimic a normal testis was developed by our team and submitted for patenting in China. The prosthesis was produced in different sizes to imitate the normal testis of the patient. To investigate the effects and safety of the testicular prosthesis, 20 patients receiving testicular prosthesis implantation were recruited for this study. Follow-up after 6 months revealed no complications in the patients. All the patients answered that they were satisfied with their body image and the position of the implants, 19 patients were satisfied with the size and 16 patients were satisfied with the weight. These results show that the testicular prosthesis used in this study can meet patient's expectations. Patients undergoing orchiectomy should be offered the option to receive a testicular prosthesis implantation. The dimensions and weight of the available prosthetic implants should be further addressed to improve patient satisfaction.

Asian Journal of Andrology (2011) 13, 903-904; doi:10.1038/aja.2011.87; published online 19 September 2011

Keywords: orchiectomy; silastic prosthesis; testicular prosthesis

\section{INTRODUCTION}

Testicular loss may arise orchiectomy for torsion, cryptorchidism, trauma, infection or malignancy. The lack of testes is considered a source of psychological trauma. An investigation of 1173 testicular cancer patients showed that $32 \%$ of these men currently or previously missed their removed testicle(s) and that $26 \%$ currently or previously had feelings of uneasiness or shame about their body because of the removed testicle(s). ${ }^{1}$ The first prosthesis, inserted in 1941, was composed of vitallium, which is an alloy of cobalt, chromium and molybdenum. ${ }^{2}$ With advancements in materials technology, the composition of testicular prostheses changed substantially. However, all the materials, including glass marbles, Gelfoam, ${ }^{3}$ plexiglass, Dacron and polyethylene, exhibited defects in biological safety or unsatisfactory aesthetics.

Solid silicone has been the most commonly used material for implantation surgery in recent decades. However, Robinson et al. ${ }^{4}$ analyzed silicone breast implants removed from 300 patients and found that $64 \%$ of the patients exhibited certain form of device disruption. In a study of 25 patients, silicone particles were found in 18 of 25 operative tissue specimens and four specimens were obtained from regional lymph nodes. ${ }^{5}$ This phenomenon of silicone migration is also known as 'gel bleed'. As a consequence of gel bleed, saline-filled prostheses replaced silicone devices in 1995. A study including 149 patients with a follow-up period of 1 year showed that saline-filled prostheses were safe and well tolerated in short-term applications. ${ }^{6}$

Until now, inadequate attention has been dedicated to the study of testicular prostheses, especially in China. The development of an effective testicular prosthesis in China could solve the cosmetic problems associated with testis removal and help resolve psychological trauma associated with the procedure.

\section{MATERIALS AND METHODS}

Development of the testicular prosthesis

Our team collaborated with the Shanghai Yihong Science and Technology Company (Shanghai, China) to design our testicular prosthesis made of medical silastic. We submitted a patent application for this testicular prosthesis, and it was approved by the Chinese State Intellectual Property Office (201020183234.1).

We manufactured the testicular prosthesis with silastic. The prosthesis is elliptical in shape, which is consistent with the anatomical shape of human testes. The prostheses were made in different sizes to meet the requirements of different patients (Table 1). Two holes were placed on both ends of the prosthesis to be used for suturing and affixing the prosthesis. To fulfil the demand for more natural-feeling implants, the prostheses were hollow instead of filled with gel. The prosthesis was tested by the China State Food and Drug Administration and was approved (Y2008011502) (Figure 1).

\section{Patients and methods}

From April 2009 to January 2011, 20 patients (average age: 28.0 \pm 4.2 years) who had undergone orchiectomy and received a testicular prosthesis implantation were recruited for this study according to the inclusion and exclusion criteria. These 20 patients included five cases undergoing treatment for prostate cancer following castration, nine cases of testicular cancer, five cases of cryptorchidism and one case of bilateral anorchia. The size of the testicular prosthesis used depended 
Table 1 The weight of different sizes of testicular prosthesis

\begin{tabular}{lcc}
\hline Volume $(\mathrm{ml})$ & Length $\times$ width $(\mathrm{cm})$ & Weight $(\mathrm{g})$ \\
\hline 2 & $2.15 \times 1.33$ & 3 \\
4 & $2.73 \times 1.67$ & 4 \\
8 & $3.43 \times 2.11$ & 7 \\
12 & $3.81 \times 2.35$ & 10 \\
15 & $4.21 \times 2.61$ & 11 \\
20 & $4.64 \times 2.87$ & 12 \\
25 & $5.00 \times 3.09$ & 14 \\
30 & $5.32 \times 3.28$ & 16 \\
\hline
\end{tabular}

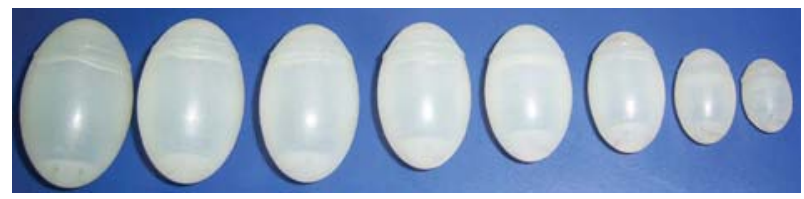

Figure 1 Varying sizes of testicular prostheses to meet the needs of different patients.

on the availability of space in the scrotum and the dimensions of the contralateral native testis or the testis that had been removed. The average operative time was $24 \mathrm{~min}$ (range: $15-30 \mathrm{~min}$ ), and all patients stayed overnight in the hospital for observation. The procedure and technique were explained to each patient in detail before the surgery. All of the patients were aware of the surgical risks and the possibility of postoperative complications. Additionally, all patients signed consent forms. We received approval for this clinical trial from the Renji Hospital Ethics Committee (Figure 2).

An anonymous questionnaire was sent to the patients by post (Supplementary Table 1) Questions addressed certain technical and psychological aspects of the procedure 6 months after the surgery, including size, weight, shape, position and degree of satisfaction.

\section{RESULTS}

No intraoperative complications were found in the 20 patients who received prostheses. A follow-up period over 6 months, with the longest duration of 12 months after the procedure, revealed that all patients were very satisfied with the position and shape of the prostheses, and no 'high riding' implants were found. Sixteen patients were satisfied with the weight of their prosthesis, and 19 patients were satisfied with the size. No postoperative complications, foreign body reactions or reoperations were reported (Supplementary Table 2).

\section{DISCUSSION}

With both increased self-awareness and societal concern with body image, the development of a testicular prosthesis to maintain a more normal cosmetic appearance to the genitalia after orchiectomy has become an important issue all over the world. The absence of a testis in a male is a psychological trauma similar to the absence of a breast or a uterus in a female. ${ }^{7}$ Testicular prosthesis implantation is a procedure that has been performed for almost 70 years. With advances in technology, the prosthesis material has changed from metal to silastic. ${ }^{1}$ There are many kinds of prostheses, but none are made in China. The implant mentioned here is the first testicular prosthesis that was designed, manufactured and used exclusively in China.

The testicular prosthesis we designed was safe, reliable, affordable and easy to use (Supplementary Figure 1). Silastic provides better chemical stability and excellent histocompatibility. This silastic prostheses did not elicit inflammatory or hypersensitivity reactions. Currently, the longest duration of follow-up is 12 months. The

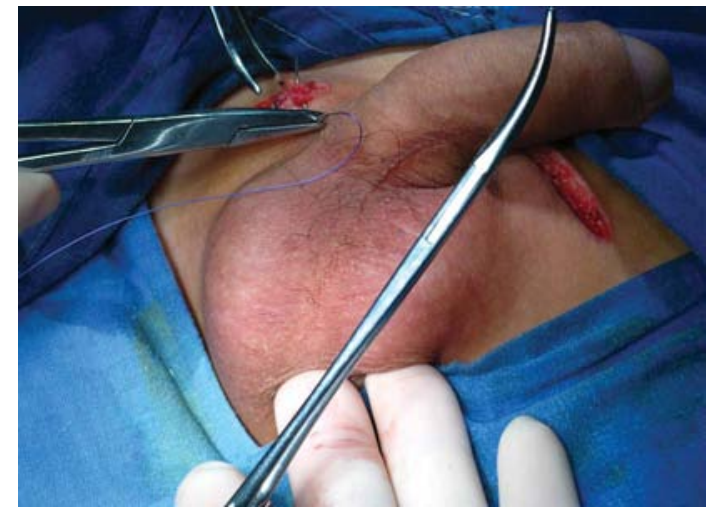

Figure 2 The external appearance of the prosthesis after implantation.

prosthesis used in this survey was free; however, when the implant is on the market, it will be much cheaper than other products manufactured by foreign companies. The procedure is easy, and a $3-\mathrm{cm}$ incision and two needles were enough for surgical fixation (Supplementary Figures 2-5). Two suture pads provide better fixation of the prosthesis and reduced the incidence of 'high-riding' prostheses, while the salinefilled testicular prosthesis only requires one. We can control the hardness of the hollow implants by changing the thickness of the silastic shell to create a natural feel. Additionally, it appears that our prosthesis resulted in positive feedback from the patients. Compared with the saline-filled prosthesis, the prosthesis we designed has no risk of rupture and leakage. This prosthesis may help solve cosmetic problems and resolve the psychological trauma of patients after orchiectomy (Supplementary Figure 6). Every patient receiving orchiectomy should be advised on the option of testicular prosthesis implantation.

\section{AUTHOR CONTRIBUTIONS}

ZL, HXC and PP performed surgery. YN and ZKC finished the followup and collected the information. YN was a major contributor in writing the manuscript. All authors read and approved the final manuscript.

\section{COMPETING FINANCIAL INTERESTS}

The authors declare that they have no competing financial interests.

\section{ACKNOWLEDGMENTS}

This study is supported by Shanghai Commission of Science Technology and Industry Cooperation Program (08410701700).

Supplementary information accompanies the paper on Asian Journal of Androglogy's website (http://www.nature.com/aja).

1 Skoogh J, Steineck G, Cavallin-StahI E, Wilderang U, Hakansson UK et al. Feelings of loss and uneasiness or shame after removal of a testicle by orchidectomy: a population-based long-term follow-up of testicular cancer survivors. Int J Androl 2010; 33: 1-10.

2 Girdansky J, Newman HF. Use of a vitallium testicular implant. Am J Surg 1941; 52: 514.

3 Bodiwala D, Summerton DJ, Terry TR. Testicular prostheses: development and modern usage. Ann R Coll Surg Engl 2007; 89: 349-53.

4 Robinson OG Jr, Bradley EL, Wilson DS. Analysis of explanted silicone implants: a report of 300 patients. Ann Plast Surg 1995; 34: 1-6.

5 Barrett DM, O'Sullivan DC, Malizia AA, Reiman HM, Abell-Aleff PC. Particle shedding and migration from silicone genitourinary prosthetic devices. J Urol 1991; 146: 319-22.

6 Turek PJ, Master VA. Safety and effectiveness of a new saline filled testicular prosthesis. J Urol 2004; 172: 1427-30.

7 Zilberman D, Winkler H, Kleinmann N, Raviv G, Chertin B et al. Testicular prosthesis insertion following testicular loss or atrophy during early childhoodtechnical aspects and evaluation of patient satisfaction. J Pediatr Urol 2007; 3: 461-5. 